\title{
Tetra and pentaquarks from the molecular perspective
}

\author{
E. Oset ${ }^{1, *}$, En Wang ${ }^{2}$, J.J. Xie ${ }^{3}$, L.S. Geng ${ }^{4}$, V.R. Debastiani ${ }^{1}$, J. M. Dias ${ }^{1}$, and W. H. Liang ${ }^{5}$ \\ ${ }^{1}$ Departamento de Fisica Teorica and IFIC, Centro Mixto Universidad de Valencia - CSIC, Institutos de \\ Investigacion de Paterna, Aptdo. 22085, 46071 Valencia, Spain \\ ${ }^{2}$ Department of Physics, Zhengzhou University, Zhengzhou, Henan 450001, China \\ ${ }^{3}$ Institute of Modern Physics, Chinese Academy of Sciences, Lanzhou 730000, China \\ ${ }^{4}$ School of Physics and Nuclear Energy Engineering and International Research Center for Nuclei and \\ Particles in the Cosmos, Beihang University, Beijing 100191, China \\ ${ }^{5}$ Department of Physics, Guangxi Normal University, Guilin 541004, China
}

\begin{abstract}
We present results for the analysis of the $B^{+} \rightarrow J / \psi \phi K^{+}$which shows the contribution of two resonances, the $X(4140)$ and $X(4160)$ and a cusp at the $D_{s}^{*} \bar{D}_{s}^{*}$ threshold tied to the molecular character of the $X(4160)$ resonance. In the second part we present the results for the theoretical approach to the new $\Omega_{c}$ states from the molecular perspective. In both cases we compare with results of the LHCb collaboration.
\end{abstract}

\section{Introduction}

In this talk we report on two issues of current interest: 1) the $B^{+} \rightarrow J / \psi \phi K^{+}$data at low $J / \psi \phi$ invariant masses and the $X(4140)$ and $X(4160)$ resonances [1]. 2) Molecular $\Omega_{c}$ states generated from coupled meson-baryon channels [2].

\section{The $B^{+} \rightarrow J / \psi \phi K^{+}$reaction}

The recent measurement of the $B^{+} \rightarrow J / \psi \phi K^{+}$reaction at $\mathrm{LHCb}[3,4]$ and analysis of the data has brought some surprises as the claim of several new states that couple to $J / \psi \phi$. Another surprise is that the $X(4140)$ deduced from the analysis, with quantum numbers $J^{P C}=1^{++}$, has a width $\Gamma \approx 83 \pm 24_{-14}^{+21} \mathrm{MeV}$, substantially larger than the average $15.7 \pm 6.3 \mathrm{MeV}$ of the former experiments.

In this work, we show that the low invariant $J / \psi \phi$ mass region, poorly reproduced in the fit of Refs. [3, 4], requires the contributions of a narrow $X(4140)$ and a wide $X(4160)$ resonance which couples to $J / \psi \phi$ but is mostly made by a $D_{s}^{*} \bar{D}_{s}^{*}$ molecule. As a consequence of this, the $J / \psi \phi$ mass spectrum develops a strong cusp around the $D_{s}^{*} \bar{D}_{s}^{*}$ threshold that the fit of Refs. [3, 4] cannot reproduce since this mechanism is not contemplated in the amplitude that is fitted to the data.

A thorough study of $X$ states emerging from the interaction of vector pairs, light-light (with light $\equiv \rho, \omega, \phi, K^{*}$ ), light-heavy (with heavy $\equiv D^{*}, D_{s}^{*}$ and $J / \psi$ ), and heavy-heavy, was done in Ref. [5] and several states were obtained that could be associated to known states. In particular, and relevant for the present work, a state $0^{+}\left(2^{++}\right)$at $4169 \mathrm{MeV}$ was

\footnotetext{
*e-mail: oset@ific.uv.es
} 


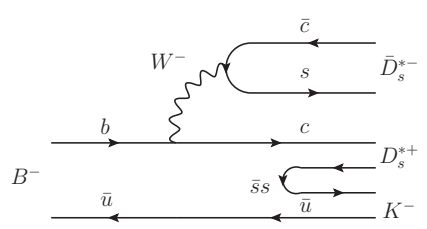

Figure 1. Microscopic quark level production of $K^{-} D_{s}^{*} \bar{D}_{s}^{*}$ in $B^{-}$ decay.

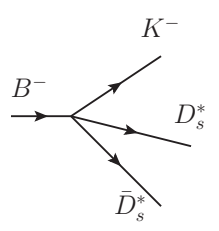

(a)

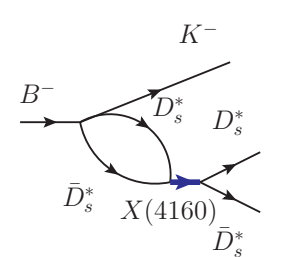

(b)

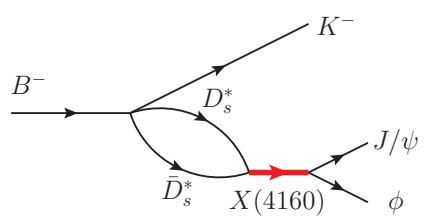

Figure 2. Mechanism for $B^{-} \rightarrow K^{-} D_{s}^{*} \bar{D}_{s}^{*}$ in the presence of the $X(4160)$ resonance.

Figure 3. Mechanism for $B^{-} \rightarrow K^{-} J / \psi \phi$ driven by the $X(4160)$ resonance.

obtained, coupling mostly to $D_{s}^{*} \bar{D}_{s}^{*}$, that qualifies as a $D_{s}^{*} \bar{D}_{s}^{*}$ molecule and was associated to the $X(4160)$, not the $X(4140)$. The dynamics used in Ref. [5] is based on the local hidden gauge approach [6], exchanging vector mesons and including contact terms. The channels included in the interaction are $D^{*} \bar{D}^{*}, D_{s}^{*} \bar{D}_{s}^{*}, K^{*} \bar{K}^{*}, \rho \rho, \omega \omega, \phi \phi, J / \psi J / \psi \omega J / \psi$, $\phi J / \psi, \omega \phi$. It was shown there that the coupling of the state to the $D_{s}^{*} \bar{D}_{s}^{*}$ was dominant $\left(g_{D_{s}^{*} \bar{D}_{s}^{*}}=18927-5524 i \mathrm{MeV}\right)$, followed by the one to $\phi J / \psi\left(g_{J / \psi \phi}=-2617-5151 i \mathrm{MeV}\right)$. The coupling to $D^{*} \bar{D}^{*}$ is $g_{D^{*} \bar{D}^{*}}=1225-490 i$, sizeable enough, that guarantees that this resonance can be seen in the $D^{*} \bar{D}^{*}$ channel. Actually, this is the channel where the $X(4160)$ was observed in the $e^{+} e^{-} \rightarrow J / \psi X, X \rightarrow D^{*} \bar{D}^{*}$ reaction [7]. The width of the $X(4160)$ is given by $\Gamma=139_{-61}^{+119} \pm 21 \mathrm{MeV}$, much wider than that of the $X(4140)$. The work of Ref. [5] gives $\Gamma=132 \pm 25 \mathrm{MeV}$. It should be noticed that with the coupling $g_{J / \psi \phi}$ obtained, one obtains a partial decay width $\Gamma_{J / \psi \phi} \approx 22 \mathrm{MeV}$. So, much of the width comes from other channels, in particular the light-light ones that have much phase space for the decay.

Next we proceed to apply our approach to the data of Refs. [3, 4] which we consider from threshold up to about $4250 \mathrm{MeV}$, above the $D_{s}^{*} \bar{D}_{s}^{*}$ threshold. The data show a narrow peak around $4140 \mathrm{MeV}$, followed by one broader structure around $4160 \sim 4170 \mathrm{MeV}$, and a remarkable cusp structure around the $D_{s}^{*} \bar{D}_{s}^{*}$ threshold. The presence of a cusp at the $D_{s}^{*} \bar{D}_{s}^{*}$ threshold in the $J / \psi \phi$ mass distribution clearly indicates a link of the resonance responsible for the $J / \psi \phi$ spectrum with the $D_{s}^{*} \bar{D}_{s}^{*}$ channel. This link can be provided assuming that the $X(4160)$ is mostly responsible for this spectrum.

The next step requires to discuss how a $D_{s}^{*} \bar{D}_{s}^{*}$ resonance is produced in the weak decay $B^{-} \rightarrow K^{-} D_{s}^{*} \bar{D}_{s}^{*}$ (we take the complex conjugate reaction to deal with $b$ quark rather than $\bar{b}$ quark). The dominant process at the quark level proceeds as shown in Fig. 1, involving external emission [8]. This allows us immediately to obtain the $B^{-} \rightarrow K^{-} D_{s}^{*} \bar{D}_{s}^{*}$ amplitude in the region around the $X(4160)$ resonance as depicted in Fig. 2. For the production of $J / \psi \phi$ with this mechanism, the tree level of Fig. 2(a) does not contribute and then we are led to the diagram of Fig. 3. 
We can then write the amplitude for the $B^{-} \rightarrow K^{-} J / \psi \phi$. The resonance $X(4160)$ obtained in Ref. [5] is a $J^{P C}=2^{++}$state with $L=0$ in $D_{s}^{*} \bar{D}_{s}^{*}$. To match angular momentum in the reaction, we need a $D$-wave in the $K^{-}$and the amplitude is thus of the type,

$$
t_{B^{-} \rightarrow K^{-} D_{s}^{*} \bar{D}_{s}^{*}}^{\text {tree }}=A\left(\vec{\epsilon} \cdot \vec{k} \vec{\epsilon}^{\prime} \cdot \vec{k}-\frac{1}{3} \vec{k}^{2} \vec{\epsilon} \cdot \vec{\epsilon}^{\prime}\right),
$$

where $\vec{\epsilon}, \vec{\epsilon}^{\prime}$ are the polarization vectors of $D_{s}^{*}$ and $\vec{D}_{s}^{*}$, and we evaluate it in the frame of reference where the $D_{s}^{*} \bar{D}_{s}^{*}$ system is at rest. The vector $\vec{k}$ is the $K^{-}$momentum in the $D_{s}^{*} \bar{D}_{s}^{*}$ rest frame. $A$ in Eq. (1) is an unknown factor that is fitted to the data.

To obtain the mass distribution for $J / \psi \phi$ through the mechanism of Fig. 3, we take the following amplitude,

$$
\begin{aligned}
A \rightarrow \quad & A \times G_{D_{s}^{*} \bar{D}_{s}^{*}}\left(M_{\mathrm{inv}}(J / \psi \phi)\right) \\
& \times t_{D_{s}^{*} \bar{D}_{s}^{*} \rightarrow J / \psi \phi}\left(M_{\mathrm{inv}}(J / \psi \phi)\right),
\end{aligned}
$$

The $G$ function appearing in Eq. (2) is the loop function for two intermediate $D_{s}^{*} \bar{D}_{s}^{*}$. To avoid potential dangers using the dimensional regularization as pointed out in Ref. [9], we use the cut off method with $q_{\max }$ fixed such as to give the same value as $G$ with dimensional regularization used in Ref. [5] at the pole position.

The amplitudes appearing in Eq. (2) are given in terms of $g_{D_{s}^{*} \bar{D}_{s}^{*}}$ and $g_{J / \psi \phi}$ obtained in Ref. [5] by means of a Breit Wigner with Flatté effect from the $D_{s}^{*} \bar{D}_{s}^{*}$ threshold incorporated.

To account for the production of $J / \psi \phi$ via the $1^{++} X(4140)$ resonance, we take the suitable operator with the kaon in $P$-wave $\left(\vec{\epsilon}_{J / \psi} \times \vec{\epsilon}_{\phi}\right) \cdot \vec{k}$, and the mass distribution coming from this source is given again with a Breit Wigner form with a weight B. Here we take $M_{X(4140)}=$ $4135 \mathrm{MeV}$, since this is the peak of the $X(4140)$ structure used in Refs. [3, 4].

The freedom to fit the data are the parameters $A, B$, and $\Gamma_{0}$, the width of the $X(4160)$ coming from decay to light vector channels. A suitable fit to the data is obtained as shown in Fig. 4 with $\Gamma_{0}=67.0 \pm 9.4 \mathrm{MeV}$ (at $68 \%$ confidence-level), which together with $\Gamma_{J / \psi \phi} \simeq 22.0 \mathrm{MeV}$ would provide $\Gamma_{X(4160)} \simeq 89.0 \pm 9.4 \mathrm{MeV}$ which is compatible with the experimental width from the PDG of $\Gamma=139_{-61}^{+111} \pm 21 \mathrm{MeV}$. As we can see in the figure, we obtain a contribution from the $X(4140)$ (blue dotted curve) that is dominant at low invariant masses, and is responsible for the peak observed in the experiment around $4135 \mathrm{MeV}$. The $X(4160)$ (green dashed curve) is responsible for most of the strength and produces a broader peak around $4170 \mathrm{MeV}$ (we take the mass $M_{X(4160)}=4169 \mathrm{MeV}$ as obtained in Ref. [5]). And finally a cusp appears at the $D_{s}^{*} \bar{D}_{s}^{*}$ threshold as it shows up in the experiment. This cusp comes from the factor $G_{D_{s}^{*} \bar{D}_{s}^{*}}\left(M_{\text {inv }}\right)$ and reflects the analytical structure of this function with a discontinuity of the derivative at threshold. One must stress that this factor appears here as a consequence of the $D_{s}^{*} \bar{D}_{s}^{*}$ molecular structure of the $X(4160)$. In an analysis like the one of Refs. [3, 4], where a sum of amplitudes for resonance excitation and some background are fitted to the data, this factor is not considered, and as a consequence the cusp around $D_{s}^{*} \bar{D}_{s}^{*}$ in the data is missed in the fit.

We have conducted the same fit using $M_{X(4140)}=4140 \mathrm{MeV}$ (pink dash-dotted curve in Fig. 4), and the fit is acceptable but slightly worse in the first two points of the spectrum.

\section{$3 \Omega_{c}$ states}

In Ref. [10] the LHCb collaboration reported five new narrow $\Omega_{c}^{0}$ states studying the $\Xi_{c}^{+} K^{-}$ mass spectrum produced in high energy $p p$ collisions: $\Omega_{c}(3000), \Omega_{c}(3050), \Omega_{c}(3066)$, $\Omega_{c}(3090)$ and $\Omega_{c}$ (3119). Predictions for such states and related ones had been done within 


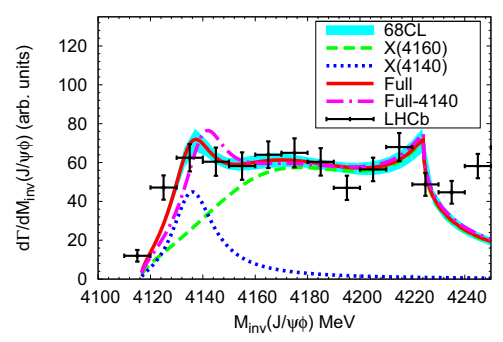

Figure 4. The $J / \psi \phi$ mass distribution of the $B^{-} \rightarrow K^{-} J / \psi \phi$ decay. The band comes considering the errors in $A, B$ and $\Gamma_{0}$ from the fit, and represents the $68 \%$ confidence-level.

quark models. Molecular states have also been used to make predictions in Refs. [11, 12] studying the interaction of coupled channels, one of them the $\Xi_{c}^{+} K^{-}$where the recent $\mathrm{LHCb}$ states were found. A more updated study along these lines was done in Ref. [13], where predictions for charmed and strange baryons are done using an interaction based on SU(6) flavor-spin symmetry in the light quark sector and SU(2) spin symmetry in the heavy quark sector, extending the SU(3) Weinberg-Tomozawa interaction. All these works take the coupled channels of meson-baryon that couple to the desired baryon quantum numbers and use a unitary scheme to obtain the scattering matrix between the channels, looking for poles of this matrix. The differences come from the input interaction and the way that loops are regularized.

In the molecular picture, an update of the work of Ref. [12] was done in Ref. [14] using some information from the experimental spectrum to regularize the loops and then giving a description of the mass and width of two states of Ref. [10] as $J^{P}=1 / 2^{-}$meson-baryon molecular states.

In the present work we shall follow Refs. $[13,14]$ for the coupled channels and the unitarization procedure. We differ in the input for the interaction, which in our case is based on the local hidden gauge approach, exchanging vector mesons $[6,15]$.

Fortunately, the exchange of $D^{*}$ is penalized with respect to the exchange of light vector mesons by a factor of $\left(\frac{m_{\rho}}{m_{D^{*}}}\right)^{2}$, which is a small factor, and then one is only introducing uncertainties in some non diagonal terms which are very small. This means we only exchange light vectors and the heavy quarks are spectators. Formally one can use the SU(4) extrapolation of the local hidden gauge approach for the meson vertices and for the diagonal terms the framework automatically filters the exchange of light vectors, providing the results that one obtains from the mapping of SU(3) to the present case. This is what is done in Ref. [14].

In the present work the diagonal terms that we evaluate coincide with those of Ref. [14] where the model of Ref. [11] is used implementing also the exchange of vector mesons and SU(4) symmetry for mesons and baryons. We, instead, use explicit wave functions for the baryon states imposing flavor-spin symmetry on the light quark sector and singling out the heavy quarks. Hence, in the baryon sector we are not using SU(4) symmetry. As a consequence, some nondiagonal terms obtained here are different from those of [14].

In addition to the work of Ref. [14] we also include pseudoscalar-baryon $\left(3 / 2^{+}\right)$components and we obtain two more states. We can identify two states of $J^{P}=1 / 2^{-}$and one of $J^{P}=3 / 2^{-}$with the states found in Ref. [10]. We also look for vector-baryon states and find three states at higher energies.

For the $1 / 2^{-}$states we take the coupled channels of Table 1 , and use the standard Lagrangians for the PPV, VVV and VBB vertices, with P pseudoscalar, V vectors and B baryon. The wave functions of the charmed baryon states are obtained considering the charm quarks 
as spectators and imposing the antisymetry on the light quarks. The Bethe Salpeter equation is then used in coupled channels.

\section{Results for $\Omega_{c}$ states}

We use the potential mentioned above and the on-shell factorized Bethe-Salpeter equation $[16,17]$

$$
T=[1-V G]^{-1} V
$$

with $G$ the meson-baryon loop function. We choose to regularize it with the cutoff method.

We look for poles in the second Riemann sheet, where we define $G_{l}^{I I}$ for $\operatorname{Re}(\sqrt{s})$ bigger than the threshold of the $l$ channel as

$$
G_{l}^{I I}=G_{l}^{I}+i \frac{2 M_{l} q}{4 \pi \sqrt{s}} ; \quad q=\frac{\lambda^{1 / 2}\left(s, m_{l}^{2}, M_{l}^{2}\right)}{2 \sqrt{s}}, \text { and } \operatorname{Im}(\mathrm{q})>0,
$$

In addition, we evaluate the couplings $g_{i}$ of the states obtained to the different channels defined such that, close to the pole $M_{R}$ we have

$$
T_{i j}=\frac{g_{i} g_{j}}{\sqrt{s}-M_{R}}
$$

and we also evaluate $g_{i} G_{i}$, which for $s$-wave gives the strength of the wave function at the origin [18].

We can see that we always get two states in the range of the masses observed experimentally. The strategy followed in these calculations is to fine tune the cutoff to adjust the pole position to some experimental data. We see that if we take $q_{\max }=650 \mathrm{MeV}$ the results agree well with the second and fourth resonances reported in Ref. [10], $\Omega_{c}(3050)$ and $\Omega_{c}(3090)$. Fitting one resonance is partly merit of fine tuning the cutoff, but then the second resonance and the widths are genuine predictions of the theory. Note that the widths are respectively $0.88 \mathrm{MeV}$ and $10.24 \mathrm{MeV}$ which agree remarkably well with the experiment, $0.8 \pm 0.2 \pm 0.1$ $\mathrm{MeV}$ and $8.7 \pm 1.0 \pm 0.8 \mathrm{MeV}$, respectively. It is instructive to see the origin of the widths. For this we look at Table 1 for the couplings to the different channels. We can see that for the lower state at $3054 \mathrm{MeV}$ only the $\Xi_{c} \bar{K}$ state is open for decay, precisely the channel where it has been observed, and the coupling of the state to this channel is very small. However, for the state at $3091 \mathrm{MeV}$ the $\Xi_{c}^{\prime} \bar{K}$ channel is also open, and the coupling to this channel is considerable. Furthermore, the coupling to $\Xi_{c} \bar{K}$ is bigger than before and there is more phase space for decay.

Table 1. The coupling constants to various channels for the poles in the $J^{P}=1 / 2^{-}$sector, with

$$
q_{\max }=650 \mathrm{MeV} \text {, and } g_{i} G_{i}^{I I} \text { in } \mathrm{MeV} \text {. }
$$

\begin{tabular}{cccccccc}
\hline \hline $\mathbf{3 0 5 4 . 0 5}+\mathbf{i 0 . 4 4}$ & $\Xi_{c} \bar{K}$ & $\Xi_{c}^{\prime} \bar{K}$ & $\Xi D$ & $\Omega_{c} \eta$ & $\Xi D^{*}$ & $\Xi_{c} \bar{K}^{*}$ & $\Xi_{c}^{\prime} \bar{K}^{*}$ \\
\hline$g_{i}$ & $-0.06+i 0.14$ & $\mathbf{1 . 9 4}+\mathbf{i 0 . 0 1}$ & $-2.14+i 0.26$ & $1.98+i 0.01$ & 0 & 0 & 0 \\
$g_{i} G_{i}^{I I}$ & $-1.40-i 3.85$ & $\mathbf{- 3 4 . 4 1}-\mathbf{i 0 . 3 0}$ & $9.33-i 1.10$ & $-16.81-i 0.11$ & 0 & 0 & 0 \\
\hline \hline $\mathbf{3 0 9 1 . 2 8}+\mathbf{i 5 . 1 2}$ & $\Xi_{c} \bar{K}$ & $\Xi_{c}^{\prime} \bar{K}$ & $\Xi D$ & $\Omega_{c} \eta$ & $\Xi D^{*}$ & $\Xi_{c} \bar{K}^{*}$ & $\Xi_{c}^{\prime} \bar{K}^{*}$ \\
\hline$g_{i}$ & $0.18-i 0.37$ & $0.31+i 0.25$ & $\mathbf{5 . 8 3 - i \mathbf { 0 . 2 0 }}$ & $0.38+i 0.23$ & 0 & 0 & 0 \\
$g_{i} G_{i}^{I I}$ & $5.05+i 10.19$ & $-9.97-i 3.67$ & $\mathbf{- 2 9 . 8 2}+\mathbf{i 0 . 3 1}$ & $-3.59-i 2.23$ & 0 & 0 & 0 \\
\hline \hline
\end{tabular}

In Ref. [2] we also look for the states of $J^{P}=3 / 2^{-}$from the pseudoscalar-baryon $\left(3 / 2^{+}\right)$ interaction. If we choose the same cutoff as in the $J^{P}=1 / 2^{-}$sector we find three states, one of them with a mass of $3125 \mathrm{MeV}$ and zero width for the lowest state, which could 
correspond to the experimental state $\Omega_{c}(3119)$.

One of us, E.O. wishes to acknowledge the support from the Chinese Academy of Science in the Program of CAS president's International Fellowship for visting scientists. This work is partly supported by the National Natural Science Foundation of China under Grant Nos. 11475227, 11375024, $11522539,11735003,11505158,11475015$, and 11647601 . It is also supported by the Youth Innovation Promotion Association CAS (No. 2016367), the China Postdoctoral Science Foundation under Grant No. 2015M582197, the Postdoctoral Research Sponsorship in Henan Province under Grant No. 2015023, and Academic Improvement Project of Zhengzhou University. This work is also partly supported by the Spanish Ministerio de Economia y Competitividad and European FEDER funds under the contract number FIS2014-57026-REDT, FIS2014-51948-C2-1-P, and FIS2014-51948-C2-2-P, and the Generalitat Valenciana in the program Prometeo II-2014/068.

\section{References}

[1] E. Wang, J. J. Xie, L. S. Geng and E. Oset, Phys. Rev. D 97, 014017 (2018)

[2] V. R. Debastiani, J. M. Dias, W. H. Liang and E. Oset, Phys. Rev. D 97, 094035 (2018)

[3] R. Aaij et al. [LHCb Collaboration], Phys. Rev. D 95, 012002 (2017)

[4] R. Aaij et al. [LHCb Collaboration], Phys. Rev. Lett. 118, 022003 (2017)

[5] R. Molina and E. Oset, Phys. Rev. D 80, 114013 (2009)

[6] M. Bando, T. Kugo, S. Uehara, K. Yamawaki and T. Yanagida, Phys. Rev. Lett. 54, 1215 (1985)

[7] P. Pakhlov et al. [Belle Collaboration], Phys. Rev. Lett. 100, 202001 (2008)

[8] L. L. Chau, Phys. Rept. 95, 1 (1983)

[9] J. J. Wu and B. S. Zou, Phys. Lett. B 709, 70 (2012)

[10] R. Aaij et al. [LHCb Collaboration], Phys. Rev. Lett. 118, 182001 (2017)

[11] J. Hofmann and M. F. M. Lutz, Nucl. Phys. A 763, 90 (2005)

[12] C. E. Jimenez-Tejero, A. Ramos and I. Vidana, Phys. Rev. C 80, 055206 (2009)

[13] O. Romanets, L. Tolos, C. Garcia-Recio, J. Nieves, L. L. Salcedo and R. G. E. Timmermans, Phys. Rev. D 85, 114032 (2012)

[14] G. Montaña, A. Feijoo and À. Ramos, Eur. Phys. J. A 54, 64 (2018)

[15] H. Nagahiro, L. Roca, A. Hosaka and E. Oset, Phys. Rev. D 79, 014015 (2009)

[16] J. A. Oller and E. Oset, Phys. Rev. D 60, 074023 (1999)

[17] J. A. Oller and U. G. Meißner, Phys. Lett. B 500, 263 (2001)

[18] D. Gamermann, J. Nieves, E. Oset and E. Ruiz Arriola, Phys. Rev. D 81, 014029 (2010) 